Daniel Weidner · Sigrid Weigel (Hrsg.)

Benjamin-Studien 1 

Daniel Weidner · Sigrid Weigel (Hrsg.)

Benjamin-Studien 1 
Bibliografische Information der Deutschen Nationalbibliothek

Die Deutsche Nationalbibliothek verzeichnet diese Publikation in der Deutschen Nationalbibliografie; detaillierte bibliografische Daten sind im Internet über http://dnb.d-nb.de abrufbar.

Alle Rechte, auch die des auszugsweisen Nachdrucks, der fotomechanischen Wiedergabe und der Übersetzung, vorbehalten. Dies betrifft auch die Vervielfältigung und Übertragung einzelner Textabschnitte, Zeichnungen oder Bilder durch alle Verfahren wie Speicherung und Übertragung auf Papier, Transparente, Filme, Bänder, Platten und andere Medien, soweit es nicht $\$ \$ 53$ und 54

URG ausdrücklich gestatten.

(C) 2008 Wilhelm Fink Verlag, München

(Wilhelm Fink GmbH \& Co. Verlags-KG, Jühenplatz 1, D-33098 Paderborn)

Internet: www.fink.de

Layout: Marietta Damm, Zentrum für Literatur- und Kulturforschung Berlin Einbandgestaltung: Evelyn Ziegler, München

Printed in Germany

Herstellung: Ferdinand Schöningh GmbH \& Co KG, Paderborn

ISBN 978-3-7705-4637-4 


\section{Benjamin, der Schreiber. Überlieferungskritische Überlegungen am Beispiel von Ausgraben und Erinnern}

\section{I.}

Walter Benjamins Schreiben ist an einen keineswegs zufälligen Umgang mit Materialien gebunden, die man für gewöhnlich bei der Untersuchung des schriftstellerischen oder philosophischen Schreibprozesses für äußerlich und sekundär betrachtet. Allerdings werden das Papier - sein Format, seine Farbe, seine Beschaffenheit -, das Schreibwerkzeug - seine Sperrigkeit, seine Gefügigkeit, sein Eigenwille -, der Schreibort - im Freien, in der Stube, am Schreibtisch - oder sogar die körperlichen Befindlichkeiten des Schreibers in der Literatur der Moderne gerade in ihrer spezifischen Materialität von primärer Wichtigkeit. Dies gilt in dem Maße, in dem sie zum Gegenstand von poetischen oder poetologischen Reflexionen und Experimenten avancieren, die sich nach Roland Barthes als »intransitives Schreiben« kennzeichnen lassen. Der moderne Schriftsteller schreibt nicht mehr etwas, sondern er schreibt, absolut. Seine Tätigkeit wird demnach losgelöst vom allfälligen Ergebnis des Schreibens betrachtet, sie bleibt primär an seine körperliche und materiale Realisation gebunden. ${ }^{1}$

Die Schreibprozessforschung spricht in diesem Falle auch von Papierarbeitern - im Gegensatz zu den Kopfarbeitern. Für letztere meint das Schreiben eigentlich einen mentalen Vorgang, der im Kopf abgeschlossen wird, um dann auf dem Schriftträger, wie durch eine Zauberhand, niedergeschrieben zu werden. ${ }^{2}$ Für Papierarbeiter hingegen ist das Schreiben nicht Ausdruck, sondern Realisation des Denkens. Letzteres ist demnach nicht

\footnotetext{
Roland Barthes: „Écrire, verbe intrasitif? (1970), in: ders.: Le bruissement de la langue. Essais critiques IV, Paris (Seuil) 1984, S. 21-31. Vgl. zum Begriff der "Skription« ders.: "Variations sur l'écriture» (1973), in: ders.: Euvres complètes. Tome II: 1966-1973. Edition établie et présentée par Éric Marty, Paris (Seuil) 1994, S. 1535-1574. Dt. Übersetzung: Roland Barthes: Variationen über die Schrift, aus dem Französischen übertragen von Hans-Horst Henschen, mit einem Nachwort von Hanns-Josef Ortheil, Mainz (Dietrich'sche Verlagsbuchhandlung) 2006.

2 Vgl. Almuth Grésillon: „Über die allmähliche Verfertigung von Texten beim Schreiben«, in: Wolfgang Raible (Hg.): Kulturelle Perspektiven auf Schrift und Schreibprozesse, Tübingen (Narr) 1995, S. 1-36. Eine systematische Einfüh-
} 
notwendig die Voraussetzung für ersteres, es muss ihm nicht vorgängig, sondern kann ihm auch nachträglich sein. Für Papierarbeiter sind die willigen Schreibgeräte, das gute Papier, die günstigen Begleitumstände kurzum das reibungslose Schreiben nicht der Normalfall, sondern das "Arkadien der Schriftstellerei«, so hat es Benjamin in der 1928 erschienenen Einbahnstraße genannt (GS IV, 113). Denn es gibt Störungen, Stockungen und Widerstände - gerade bei den Papierarbeitern. Das Schreiben scheint sich vornehmlich dort bei sich selbst aufzuhalten, wo es nicht reibungslos funktioniert und wo es sich regelrecht in seine Bestandteile auflöst. Die Hand, das Schreibinstrument und der Kopf können in Widerstreit geraten und gehen bisweilen ihre eigenen Wege. ${ }^{3}$ Das Resultat sind in der Regel Schriftträger, die mit Streichungen, Überschreibungen, Einschüben, Nachträgen u.ä. übersäht sind und an denen das Ringen um eine definitive Gestalt sichtbar ist.

Eine solche materiale Poetik entspricht Benjamins zahlreichen Äußerungen zum Schreiben und zu seiner eigenen Schreibpraxis durchaus. "Die Technik des Schriftstellers in dreizehn Thesen«, publiziert im Rahmen der Einbahnstraße, bildet eine kondensierte und stilisierte Fassung dieses Programms (GS IV, 106f.). Alle Bereiche der Schreibtätigkeit sind von Benjamin in ihrer Materialität thematisiert und in der Praxis bedacht worden. ${ }^{4}$ Seine eigene Produktion ist eine dezidiert handschriftliche gewesen, die sich, medienhistorisch reflektiert, vom Gebrauch der Schreibmaschine fern gehalten und in Abgrenzung zur typisierten Schrift bestimmt hat. Daher rührt die penible Aufmerksamkeit für den Füllfederhalter, dessen »Tyrannei« Benjamin auch beklagt, für Qualität und Farbe des Papiers, das er kleinformatig bevorzugt, für die Schreibfläche, die er bisweilen mit einer möglichst kleinen Schrift gestaltet hat. ${ }^{5}$ Daher rührt auch sein Interesse an der Äußerlichkeit von Schrift, das etwa in seinen Reflexionen auf Schriftbildlichkeit, Graphologie und Kalligraphie zum Ausdruck gekommen ist. Aber auch der körperliche Vollzug im Schreibakt, wie er in der Praxis des Abschreibens - eine Tätigkeit, der er bei der Entstehung seiner Texte

rung bietet dieselbe Autorin unter dem Titel Literarische Handschriften. Einführung in die "critique génétique», Bern u.a. (Peter Lang) 1999.

3 Vgl. Martin Stingelin: "Schreiben`. Einleitung«, in: ders. (Hg.): "Mir ekelt vor diesem tintenklecksenden Säkulum «. Schreiben im Zeitalter der Manuskripte, München (Fink) 2004, S. 7-18. Stephan Kammer: »Reflexionen der Hand", in: Davide Giuriato/ders. (Hg.): Bilder der Handschrift. Die graphische Dimension der Literatur, Basel - Frankfurt a.M. (Stroemfeld/Nexus) 2006, S. 131-161.

4 Vgl. für eine anschauliche Dokumentation dieser Materialität: Walter Benjamins Archive. Bilder, Texte und Zeichen, hg. v. Walter Benjamin Archiv, Frankfurt a.M. (Suhrkamp) 2006. Vgl. zusammenfassend: Erdmut Wizisla: "Verzettelte Schreiberei«, in: Sinn und Form (2006), S. 266-273 bzw. in: Trajekte. Zeitschrift des Zentrums für Literatur- und Kulturforschung Berlin 13 (2006), S. 8-12.

5 Vgl. Davide Giuriato: "(Mechanisiertes) Schreiben«, in: ders./Martin Stingelin/Sandro Zanetti (Hg.): "SCHREIBKUGEL IST EIN DING GLEICH MIR: VON EISEN". Schreibszenen im Zeitalter der Typoskripte, München (Fink) 2005, S. 7-20. 
selbst methodisch nachgegangen ist - in den Vordergrund tritt, ist ihm ein Vorzug des Handschriftlichen gegenüber dem Maschinenschriftlichen gewesen.

Der Übergang in Druck und Typographie, den er - wie die Drucklegung der Einbahnstraße und des Trauerspiel-Buchs bezeugen - mit großer Aufmerksamkeit verfolgt hat, muss er als eine Schwelle empfunden haben, die er nicht in jedem Fall zu überschreiten für nötig erachtet hat. So hält er etwa für die Lehre vom Ähnlichen fest: »Drucken lasse ich besagte Blätter nicht, ja ob sie auch nur einer Maschinenübertragung fähig sind, erscheint mir noch nicht ganz sicher.« (GB IV, 163) Wenn ein großer Teil von Benjamins Schriften in Manuskriptform überliefert ist, so ist das nicht nur auf eingeschränkte Publikationsmöglichkeiten zurückzuführen, sondern auch auf ihren textuellen Status, den Benjamin offenbar sehr genau von demjenigen publizierter Schriften unterschieden hat. ${ }^{6}$

Diese Eigentümlichkeit des Handschriftlichen, die bei der Übersetzung in Druckschrift verloren geht, hat schon Theodor W. Adorno mit Bezug auf die Briefe Benjamins bemerkt. Dass dabei Schriftträger und Schreibmittel nicht zufällig zum Einsatz gekommen sind, erklärt er sich damit, dass Benjamin "der physische Akt des Schreibens Lust» bereitet habe. ${ }^{7}$ Diese Beobachtung - davon gehen die folgenden Ausführungen aus - muss auch für die anderen Bereiche von Benjamins Produktion Geltung haben. Seine materiale Poetik kann nicht auf idiosynkratisch anmutende Praktiken des Briefschreibens reduziert werden, sondern bestimmt weit grundlegender Gehalt und Gestalt seiner Schriften. ${ }^{8}$

Die Tatsache, dass Benjamins Schreiben in vielen Fällen zu keiner abgeschlossenen Gestalt geführt und sich nicht immer zu einem `Text` gefügt hat, erfordert dringend eine produktionsästhetische Perspektive, die das Augenmerk mit Entschiedenheit auf die Entstehungsprozesse lenkt. In diesem Punkt kann die Benjamin-Forschung Neuland betreten. Was damit zudem zur Diskussion steht, ist nichts Geringeres als eine überlieferungsgeschichtliche Revision ihrer textuellen Grundlage. Was heute als Texte Benjamins gelesen werden kann, ist das Resultat von jahrzehntelanger Werkpolitik. ${ }^{9}$ Sie unterliegen einer

6 Auch dies ist ein gewichtiger Aspekt, den eine Edition sichtbar machen sollte, um der Forschung ein verlässliches Instrument in die Hand zu geben. Man könnte hier mit relativ einfachen Mitteln, etwa mit einer klaren und konsequenten editorischen Einteilung in publizierte Schriften einerseits und Schriften aus dem Nachlass anderseits Benjamins Werkstruktur, die keine zufällige und unkalkulierte Werkstruktur ist, deutlicher zum Vorschein bringen.

7 Theodor W. Adorno: „Benjamin, der Briefschreiber" (1966), in: ders.: Noten zur Literatur, Frankfurt a.M. (Suhrkamp) 71998, S. 585. Vgl. weiterführend: Klaus Garber: Walter Benjamin als Briefschreiber und Kritiker, München (Fink) 2005, bes. S. $36 f$.

8 Vgl. zu einer systematischen Beschreibung dieser Poetik: Davide Giuriato: Mikrographien. Zu einer Poetologie des Schreibens in Walter Benjamins Kindheitserinnerungen (1932-1939), München (Fink) 2006, bes. S. 27-59.

9 Vgl. Detlev Schöttker: "Edition und Werkkonstruktion. Zu den Ausgaben und Schriften Walter Benjamins«, in: Zeitschrift für Deutsche Philologie 116 (1997), S. 294-315. Ausführlicher zur Überlieferung von Benjamins Schriften ders.: Konstruktiver Fragmentarismus, Frankfurt a.M. (Suhrkamp) 1999. 


\section{DAVIDE GIURIATO}

editorisch hergestellten Suggestion von Abgeschlossenheit, die sie aber in der Logik von Benjamins Produktion häufig nicht besitzen. Alles, was aus dem Nachlass an Aufzeichnungen, Notizen, Entwürfen, Arbeitshandschriften überliefert ist und in den Archiven schlummert, ist vielmehr Zeuge eines infiniten work in progress, der sich nicht in ein werkästhetisches Korsett zwingen lässt. Im Folgenden wird an einer kleinen, aber prominenten Aufzeichnung Benjamins materiale Poetik des Schreibens exemplarisch veranschaulicht. Die Fragen nach den Implikationen und Konsequenzen für den interpretatorischen und überlieferungskritischen Umgang mit seinen nachgelassenen Schriften stehen dabei im Vordergrund.

\section{II.}

Benjamins Aufzeichnung Ausgraben und Erinnern aus dem Jahre 1932 gehört zu den kanonischen Texten einer abendländischen Theoriegeschichte des Gedächtnisses. ${ }^{10}$ In den vergangenen dreißig Jahren ist ihr eine beachtliche Zahl an Kommentaren und Interpretationen gewidmet worden. ${ }^{11}$ Sei es als kondensierter Vorspann zu Benjamins Geschichtsphilosophie, sei es als verdichteter Abriss seiner Erinnerungs- und Gedächtnistheorie, sei es als Lektüre- und Schreibanleitung zu seinem eigenen Erinnerungswerk, der Berliner Kindheit - der Aufzeichnung wurde durchweg eine in sich gegründete Abgeschlossenheit und Gültigkeit unterstellt, die ihren programmatischen Status bekräftigte.

Die Rezeptionsgeschichte dieses Textes ist grundlegend durch die editorische Geschichte von Benjamins Schriften bedingt, die postum ab 1955 mit Adornos Edition der Schriften in zwei Bänden eingesetzt hat. Gut zwei Jahrzehnte später ist Ausgraben und Erinnern im Rahmen der Gesammelten Schriften wie ein in sich gefügter und kontextunabhängiger Solitär in der Abteilung der nach Adorno so genannten »Denkbilder« konstituiert und

10 Vgl. Uwe Fleckner: Die Schatzkammern der Mnemosyne. Ein Lesebuch mit Texten zur Gedächtnistheorie von Platon bis Derrida, Dresden (Verlag der Kunst) 1995, S. $274 \mathrm{f}$.

11 Hier nur eine Auswahl: Christiaan L. Hart Nibbrig: "Das déjàvu des ersten Blicks. Zu Walter Benjamins Berliner Kindheit um neunzehnhundert", in: DVjs 47/4 (1973), S. 711-729; Anna Stüssi: Erinnerung an die Zukunft. Walter Benjamins "Berliner Kindheit um Neunzehnhundert", Göttingen (Vandenhoeck \& Ruprecht) 1977, S. 70f.; Josef Fürnkäs: Surrealismus als Erkenntnis: Walter Benjamin - Weimarer Einbahnstraße und Pariser Passagen, Stuttgart (Metzler) 1988, S. 117-148; Marianne Muthesius: Mythos Sprache Erinnerung. Untersuchungen zu Walter Benjamins Berliner Kindheit um neunzehnhundert, Basel - Frankfurt a.M. (Stroemfeld) 1996, S. 90-95; Sigrid Weigel: Entstellte Ähnlichkeit. Walter Benjamins theoretische Schreibweise, Frankfurt a.M. (S. Fischer) 1997, S. 27-31; Nicolas Pethes: Mnemographie. Poetiken der Erinnerung und Destruktion nach Walter Benjamin, Tübingen (Niemeyer) 1999, S. 8-14; Detlev Schöttker: "Erinnern", in: Michael Opitz/Erdmut Wizisla (Hg.): Benjamins Begriffe, Frankfurt a.M. (Suhrkamp) 2000, Bd. 1, S. 260-298, bes. S. 265f.; Dominik Finkelde: Benjamin liest Proust. Mimesislehre - Sprachtheorie - Poetologie, München (Fink) 2003, S. 150-155. 
abgedruckt worden (GS IV, 400). Der kurze Text ist entsprechend im vierten Band der Ausgabe wiedergegeben worden, in dem nach Ansicht der Editoren »abgeschlossene Texte Benjamins« stehen sollten (GS IV, 883), in dem de facto aber neben tatsächlich abgeschlossenen und publizierten Büchern wie Einbahnstraße oder Deutsche Menschen auch unabgeschlossene Bücher (wie die Berliner Kindheit) und unabgeschlossene Aufzeichnungen aufgenommen worden sind.

Mit dem Begriff »Denkbild« hat Adorno für Benjamin eine Denkform reklamiert, "die philosophischen Gehalt unter der Maske spielerischer Reflexion über unscheinbare Gegenstände« verbirgt und »die nicht sowohl dem begrifflichen Denken Einhalt gebieten als durch ihre Rätselgestalt schockieren und damit Denken in Bewegung bringen " will. ${ }^{12}$ Während der neuplatonischen Prägung des Denkbilds bei Stefan George wenig Erfolg beschieden gewesen sei, habe es als philosophische Form bei Benjamin eine neue Konzeption erfahren, die an die Stelle der platonischen Bilder "gekritzelte Vexierbilder" gesetzt habe. Benjamin selbst hat die Bezeichnung nur in einem Fall für die Publikation von sieben Kurztexten verwendet, die am 15.11.1933 in der Frankfurter Zeitung erschienen sind (GS IV, 428-433). Adorno hat damit allerdings etwas weit Umfassenderes gemeint: Er hat sie als eine für Benjamins Denken spezifisch verdichtete Form betrachtet, die auch den Blick auf die vielen anderen kurzen Texte, unter anderem etwa die aphoristischen Texte der Einbahnstraße, bestimmt. So ist mit dem Begriff "Denkbild « nach Adorno nicht nur eine Denkform charakterisiert, sondern eine eigene Textgattung geboren worden, die in der Folge die editorische Praxis bestimmte (GS IV, 883). „Denkbild« ist zur Gattungsbezeichnung für eine umfangreiche und heterogene Reihe von Kurztexten avanciert, die weit länger war (GS IV, 305-438), als die wenigen von Benjamin als »Denkbilder« publizierten Texte. Als genuin Benjaminsche Gattung sind die »Denkbilder« heute noch weit bekannt. ${ }^{13}$ Freilich sind diese Texte in ganz verstreuten Überlieferungszusammenhängen entstanden. Ausgraben und Erinnern beispielsweise ist von Benjamin keineswegs als »Denkbild" geschrieben oder auch nur beabsichtigt worden.

Die methodische Aufwertung des archivarischen Dokuments - so wie es im Walter Benjamin Archiv (Berlin) als Ms 929 aufbewahrt wird - führt zu einer anderen Geschichte der Aufzeichnung. ${ }^{14}$ Ausgraben und Erinnern ist bei genauerer Betrachtung ein Entwurf,

12 Theodor W. Adorno: „Benjamins ,Einbahnstraße«, in: ders.: Noten zur Literatur, Frankfurt a.M. (Suhrkamp) 71998, S. 681.

13 Begünstigt wurde diese Rezeptionsweise durch Publikationen wie: Walter Benjamin: Denkbilder, Frankfurt a.M. (Suhrkamp) 1974.

14 Im Falle von Benjamins nachgelassenen Schriften und ihrer bislang unzulänglichen Edition stellen die Walter Benjamin Archive in Berlin und Jerusalem das Apriori des Umgangs mit eben diesen Schriften dar (vgl. zum Archiv als historischem Apriori: Michel Foucault: Archäologie des Wissens, übersetzt von Ulrich Köppen, 


\section{DAVIDE GIURIATO}

der eine Genese besitzt und der keine abgeschlossene Textgestalt durch Benjamins Hand erfahren hat. Man hat es im strengen Sinne nicht mit einem Text, sondern mit der Möglichkeit eines Textes zu tun, die keine verbindliche Form besitzt, da sie von Benjamin nicht für die Veröffentlichung vorgesehen worden ist. Diese überlieferungskritische Unterscheidung ist für den interpretatorischen Umgang mit der Aufzeichnung, die im Bild der Archäologie selbst eine kleine Skizze zu einer Theorie der Überlieferung enthält, grundlegend. Um dies nachzuweisen, ist es allerdings unerlässlich, das komplexe überlieferungsgeschichtliche Gewebe, wie es aus der archivarischen Spurensuche hervorgegangen ist, quasi archäologisch, Schicht für Schicht, vorzustellen. Dabei wird gleichsam ein neuer entstehungslogischer Kontext der Aufzeichnung erkennbar, der ihren programmatischen Status einigem Zweifel aussetzt.

Eine erste, titellose Niederschrift findet sich in einem Entwurfskomplex aus dem Jahre 1932, in dem Benjamins erste Kindheitserinnerungen unter dem Titel Berliner Chronik stehen (GS VI, 486f.). Diese lose zusammenhängenden Erinnerungsabschnitte, die neben einer ganzen Reihe von autobiographischen Notaten aus Benjamins Kindheit und Jugendzeit auch einige Meta-Reflexionen zum Wesen der Autobiographie und der Erinnerung enthalten, hat er während seines Aufenthaltes auf Ibiza zwischen April und Juli 1932 in einem seiner bevorzugten kleinformatigen Notizhefte aus Pergamentpapier niedergeschrieben, ohne sie zu einem abgeschlossenen Ganzen fertig zu stellen. Vielmehr benutzte er einige Aufzeichnungen der Berliner Chronik als Vorlage, als er die Kindheitserinnerungen nach seinem Weggang von Ibiza im Spätsommer 1932 an der norditalienischen Küste umzuschreiben begann. Diese Umschriften entstanden im Hinblick auf das allmählich sich abzeichnende Projekt Berliner Kindheit um 1900. Die sehr spärlichen Informationen im Kommentarteil der Gesammelten Schriften halten fest, dass Ausgraben und Erinnern auf diese "sehr ähnlich lautende Passage" in der Berliner Chronik zurückgeht (GS IV, 1002). Was damit suggeriert wird, ist die Vorstellung, dass Benjamin den Abschnitt fast ohne Änderungen auf ein separates, für sich stehendes Blatt im Sinne einer handschriftlichen Reinschrift abgeschrieben hat. Ausgraben und Erinnern wäre demnach

\footnotetext{
Frankfurt a.M. (Suhrkamp) 71995, S. 187 und Wolfgang Ernst: Im Namen von Geschichte. Sammeln Speichern - Er/Zählen. Infrastrukturelle Konfigurationen des deutschen Gedächtnisses, München (Fink) 2003. Der kritische Rückgriff auf die Überlieferungslage, ein entstehungsgeschichtliches Verständnis seiner Schriften, die editorische Interpretation und Konstitution dieses Nachlasses müssen von einer verlässlichen archivarischen Sicherung dieses Nachlasses ausgehen können. Da keine Edition ein Archiv ersetzt, gehören die materielle Sicherung, die detaillierte Sichtung und die katalogisierte Aufnahme des Bestandes zu den dringlichsten Aufgaben der Benjamin-Archive. Erst nach dieser archivarischen Sicherung der Grundlagen kann eine Neu-Edition seiner Schriften, die historisch-kritischen Maßstäben genügen möchte, einsetzen (vgl. hierzu ausführlicher Giuriato: Mikrographien [Anm. 8], S. 209-222, bes. S. 215).
} 
ein aus seinem ursprünglichen Kontext extrapoliertes »Denkbild«, das den Weg aus den persönlich gefärbten Kindheitserinnerungen gefunden hat und nun als allgemeine Theorie des Gedächtnisses autonom da steht. Diese Sicht der Dinge ist die heute ungebrochen vorherrschende: Ausnahmslos jede Studie, die sich mit diesem Text beschäftigt, geht von dieser Prämisse aus.

Die Textgeschichte allerdings zeigt, dass Ausgraben und Erinnern einen komplexeren Status in Benjamins Produktion besitzt. Die entstehungsgeschichtliche Recherche kann belegen, dass Ausgraben und Erinnern im unmittelbaren Kontext der Berliner Kindheit niedergeschrieben worden ist. Als Benjamin im Juli 1932 Ibiza verlässt und beginnt, seine Kindheitserinnerungen der Berliner Chronik umzuschreiben, entstehen in der Zeit von August-September eine ganze Reihe von Textentwürfen, die auf je einzelnen Blättern stehen. Noch ist die Berliner Kindheit um 1900 als Buchprojekt nicht absehbar. Benjamin spricht brieflich vielmehr davon, dass er an einer losen »Folge von Aufzeichnungen [...], die frühe Erinnerungen betreffen«, arbeite (GB IV, 128f.). Aus dieser Zeit konnte eine Titelliste im Walter Benjamin Archiv gefunden werden, die die Titel dieser losen Folge von Kindheitserinnerungen enthält und die unmissverständlich bezeugt, dass Ausgraben und Erinnern nicht etwa einen für sich stehenden Text darstellt, sondern im Kontext von Benjamins Arbeit an seinen Kindheitserinnerungen entstanden ist. Auf der besagten Titelliste ist der Textentwurf unter dem Titel »Erinnern und Ausgraben « aufgeführt. ${ }^{15}$ Noch auf einer späteren Titelliste, die im Herbst 1932 als eine der ersten Listen zur Berliner Kindheit um 1900 angelegt worden ist und die Benjamin noch bis 1938 benutzt haben könnte, ist er unter dem Titel »Ausgrabungen $"$ zu finden. ${ }^{16}$

Demnach ist klar, dass Ausgraben und Erinnern nie den Rahmen der Kindheitserinnerungen verlassen hat. Vielmehr ist der Entwurf nach der Berliner Chronik als Umarbeitung eines Textabschnittes in einer Schreibphase entstanden, in der die Arbeit an der Niederschrift einzelner Textstücke auf je einzelnen Blättern voran geht, ohne dass das Projekt der Berliner Kindheit um 1900 schon deutlich erkennbar wäre. Die Titellisten bezeugen eher, dass Benjamin eine Reihe von Texten mehr oder weniger lose zu einem Plan zusammengefasst hat, den er später immer und immer wieder revidiert hat, indem er neue Stücke geschrieben und zum variablen Bestand der Berliner Kindheit hinzugenommen oder indem er ältere Stücke wieder ausgesondert und liegen gelassen hat. Ausgraben und Erinnern ist demnach, das kann nun dezidiert unterstrichen werden, kein Denkbild, sondern der Entwurf eines kleinen Stücks Kindheitserinnerung, den Benjamin später nicht in die

15 Diese Titelliste ist erstmals publiziert bei: Giuriato: Mikrographien (Anm. 8), S. 279.

16 Ebd., S. 281. 
Textsammlung der Berliner Kindheit aufgenommen und nicht als Publikation geplant hat. Demnach ist klar, dass Benjamin die Aufzeichnung ausgesondert und verworfen hat.

3.

Für die Schreibphase von August bis September 1932, also der Zeit einer akuten existentiellen Krise und des geplanten Selbstmords in Nizza, fällt auf, dass sich Benjamins Kindheitserinnerungen vermehrt um die Tätigkeit des Erinnerns selbst kümmern. Zeuge einer solchen Selbsthematisierung ist nicht nur Ausgraben und Erinnern, sondern auch ein anderes, bis vor kurzem unpubliziertes Stück, das unter dem Titel Rousseau-Insel niedergeschrieben worden ist. Das Manuskript, aus dessen zweiter Hälfte später das Textstück Zwei Blechkapellen entstanden ist, stellt übrigens ein besonders anschauliches Beispiel für Benjamins Mikrographie dar. Einem Walserschen Mikrogramm ähnlich hat man es mit einer Buchstabengröße von 1 bis $2 \mathrm{~mm}$ zu tun, und die Verteilung der Schrift auf dem nicht voll geschriebenen Blatt macht deutlich, dass Benjamins Mikrographie ihren inneren Grund nicht in papierökonomischen Voraussetzungen haben kann. ${ }^{17}$ Das Benjaminsche Mikrogramm enthält unter dem Titel Rousseau-Insel eine Reflexion auf die Struktur der eigenen Erinnerung, die sich in einer auffälligen Metaphorik kundtut. ${ }^{18}$ Benjamin rekurriert nämlich auf ein Bild, das in der Regel gerade für das Gegenteil, nämlich für das Vergessen, eingesetzt wird. ${ }^{19}$ Er nennt seine eigenen Erinnerungsbruchstücke Inseln im »Meer des Erinnerns", die er mit dem Boot seines Gedächtnisses aufsuchen muss. Entscheidend ist hier, dass Benjamin diese erinnerungspoetologische Metapher, die mehr als das Erinnerte den Vorgang des Erinnerns figuriert, in der fortschreitenden Arbeit am Stück Zwei Blech-

17 Vielmehr handelt es sich bei der Kleinstschrift um eine Variante unter vielen von Benjamins Handschrift: Von ihr sollte man vielleicht sagen, dass sie in ihrer Gesamtheit nicht den ein und einzigen, identifizierbaren Charakter besitzt. Manchmal schreibt Benjamin auch größer und flüchtiger. Mehr als von Benjamins Handschrift im emphatischen Sinne, sollte man von Benjamins Handschriften im Plural sprechen. Mehr als Ausdruck eigenhändiger und das heißt traditionell sauthentischer Zeichen, sollte man in Benjamins Handschriften einen Möglichkeitsspielraum erkennen, der es ihm etwa im Gegensatz zur Maschinenschrift erlaubte, den Schriftzug, den "Charakter", auch anders und neu zu gestalten (vgl. dazu grundlegend: Nicolas Pethes: Mnemographie [Anm. 11], S. 101f. und Heiko Reisch: Das Archiv und die Erfahrung. Walter Benjamins Essays im medientheoretischen Kontext, Würzburg [Königshausen \& Neumann] 1992, bes. S. 51).

18 Die Stelle lautet: „darum kann ich seit Jahren nicht mehr zur Rousseau-Insel und darum blieb in meinem Innern ihr Name, als wär er selber Insel darinnen, von allem Austausch mit dem Binnenlande meines Denkens abgeschnitten. Ich halte ihn auch heute nur darum auf weil kürzlich Licht auf eine ganze Inselgruppe dieser Art - unfruchtbare und unbewohnte Riffe, die unzugänglich im Meer des Erinnerns liegen, gefallen ist. Damals, vor wenigen Tagen - und das war im Pavillon d'Armonville im Gespräche mit H. machte ich in mir wie ein suchendes Boot das Gedächtnis los - das Gedächtnis, welches Verdacht hatte, es sei das kostbarste Gut meines Lebens, ohne ihm den gebührenden Zoll zu entrichten, in einem Labyrinth solcher Inseln ihm entgangen. Eine davon war in der Wohnung meiner Großmutter [...] (vgl. Giuriato: Mikrographien [Anm. 8], S. 275).

19 Vgl. Harald Weinrich: Lethe. Kunst und Kritik des Vergessens, München (C.H. Beck) 1997. 
kapellen weglässt. Ebenso wie Ausgraben und Erinnern, das ja als Ganzes aus der Berliner Kindheit ausgeschlossen und weggelassen wird, bewahrt er aber die Arbeitshandschrift unter seinen nachgelassenen Papieren auf.

Der überlieferungskritische Kontext von Ausgraben und Erinnern macht deutlich, dass Benjamins Arbeit an seinen Kindheitserinnerungen in einer Weise vorangeht, die methodische Reflexionen auf die eigene Erinnerungstätigkeit allmählich weglässt. Diese fortschreitende Aufhebung des erinnernden Ich entspricht im Übrigen einer Tendenz, die die gesamte Revisionsarbeit an den Kindheitserinnerungen bis zum späten Pariser Typoskript von 1938 charakterisiert. ${ }^{20}$ Ohne diesen Punkt weiter verfolgen zu können, steht hier allerdings die methodische Frage im Vordergrund, welche Bedeutung dies nun für das Ausgesonderte besitzt: Sind die Textentwürfe für ungültig zu erklären? Wenn ja, warum hat sie Benjamin nicht vernichtet? Wenn nein, was macht den Unterschied zu abgeschlossenen und publizierten Texten? Kurzum: Welchen Stellenwert besitzen Benjamins nachgelassene Aufzeichnungen und wie sind sie zu lesen?

Zur Beantwortung dieser Fragen sind zwei texttheoretische Prämissen entscheidend. Erstens muss festgehalten werden, dass man es bei Aufzeichnungen wie Ausgraben und Erinnern nicht mit fertigen Texten, sondern mit Textentwürfen zu tun hat, deren Status von demjenigen der Textstücke zu unterscheiden ist, die tatsächlich in die Berliner Kindheit aufgenommen und teilweise auch publiziert worden sind. Zweitens folgt daraus, dass Ausgraben und Erinnern einen denkbar ambivalenten textuellen Status besitzt, den es genauer zu beschreiben gilt.

Als Teil der Berliner Chronik bezeugt er paradigmatisch die intensive Reflexion der ersten Erinnerungen auf die Tätigkeit des Erinnerns selbst. Von ihr heißt es in Ausgraben und Erinnern zudem, dass sie sich nicht scheut, »immer wieder auf einen und denselben Sachverhalt zurückzukommen« (GS IV, 400). Die erinnerungstheoretische Überlegung, die darin enthalten ist, besagt, dass die methodisch verfolgte Wiederholung für die Erinnerungstätigkeit konstitutiv ist. Immer wieder kommt sie auf dasselbe zurück, ohne im Dienste eines abschließenden Telos zu stehen. Die Aufzeichnung fasst diese Struktur im Bild des archäologischen Forschers, der Schicht für Schicht im Erdreich des Gedächtnisses wühlt und für den die eigentlichen Sachverhalte nicht die erinnerten Inhalte, sondern die Schichten selbst sind (ebd.). Diese poetologische Reflexion auf die Prozessualität und Medialität der Erinnerungstätigkeit findet nun eine eigentümliche Entsprechung in Benjamins Schreiben selbst. Dieses ist nämlich ebenso prozessual und ohne Ende. Die

20 Vgl. dazu auch Kai Kaufmann: "Rudolf Borchardts und Walter Benjamins Berliner Kindheiten um 1900«, in: Zeitschrift für Germanistik. Neue Folge 1 (1998), S. 374-386. 
Korrespondenz zwischen Poetik und Praxis ist auf überlieferungsgeschichtlicher Ebene zu beobachten, auf der es zum Abbruch der Berliner Chronik und zum Ab- und Umschreiben der Kindheitserinnerungen kommt. Die Umschrift Ausgraben und Erinnern gewinnt also als Umschrift einen eminent selbstbezüglichen Wert. Sie verweist darauf, dass die Praktiken Schreiben, Ab- und Umschreiben für die Tätigkeit des Erinnerns vorrangig und konstitutiv sind.

Wenn in dieser Perspektive der Textentwurf Ausgraben und Erinnern als Umschrift gelesen wird, die gerade in ihrer schriftlichen Verfasstheit dokumentiert, dass für die frühen Kindheitserinnerungen Schreiben und Erinnern eine prozessuale Dynamik entfalten und zu einer tief greifenden Umarbeitung der Berliner Chronik hin zum Projekt der Berliner Kindheit führen, dann besteht sein ambivalenter Status zudem darin, dass diese Umschrift später nicht wieder aufgenommen wird. Der Entwurf wird also im Fortlauf des Schreibens verworfen und zugleich von Benjamin selbst beiseite gelegt und aufbewahrt. Sein ambivalenter Status besteht demnach auch darin, dass er in der Schwebe des Provisorischen verharrt und als unfertiges Geschöpf trotzdem archiviert wird. Benjamin hat den Textabschnitt deshalb auf ein separates Blatt ab- und umgeschrieben, weil er ihn in die Textsammlung der Berliner Kindheit aufnehmen wollte, ihn dann aber im Verlauf der Arbeit wieder aussonderte, so wie etwa die Stücke Gesellschaft, Neuer deutscher Jugendfreund, Das Pult u.a. Ebenso wie die Berliner Kindheit, die Benjamin einem ständigen Revisionsprozess unterzogen hat, ohne sie zu einem Abschluss in definitiver Buchform zu bringen, ${ }^{21}$ steht Ausgraben und Erinnern - auch hierin paradigmatisch - schwebend zwischen Festlegung und Aufhebung, zwischen Entwurf und Verwerfung, zwischen Realisation und Revision. Diese überlieferungsgeschichtliche Ambiguität, die auch die Ambiguität von Benjamins nachgelassenen Schriften insgesamt ist, gilt es ernst zu nehmen. Benjamins verzettelte Produktion ist als eine Verkettung von Möglichkeiten zu betrachten, die sich in aller Regel nicht verbindlich realisiert haben.

Ist nun vor diesem überlieferungskritischen Hintergrund klar, dass Benjamins nachgelassene Schriften nicht als abgeschlossene und in sich geschlossene Texte gelesen werden können, so muss sich das Interesse weg vom Text und hin zum Schreib- und Denkprozess verschieben. Was mit diesem Perspektivenwechsel auf Anhieb erkennbar wird, ist der Umstand, dass beispielsweise Ausgraben und Erinnern noch keine in sich gefügte Theorie des Gedächtnisses bereithält, sondern vornehmlich Benjamins theoretische Arbeit an Gedächtnis, Erinnerung und Überlieferung bezeugt. Diese Arbeit schreitet nicht so voran,

${ }^{21}$ Vgl. hierzu ausführlich: Giuriato: Mikrographien (Anm. 8), bes. S. 223-268. 
dass sie einen abschließend durchdachten Gedanken im Kopf zurechtlegt und dieser dann fein säuberlich niedergeschrieben wird, sondern vielmehr so, dass die Arbeit am Konzept noch auf dem Papier beim Schreiben stattfindet. Dies kann man am besten an Stellen beobachten, an denen einmal Gedachtes durchgestrichen und neu geschrieben wird. Aber auch der Umstand, dass ein Textentwurf weggelegt und nicht fertig geschrieben wird, kann darauf hinweisen, dass ein Konzept nicht zu Ende gedacht oder dass Abstand von ihm genommen worden ist. Genau dies ist auch im Fall von Ausgraben und Erinnern geschehen, wie nun im Folgenden durch eine genauere Analyse des Schreibprozesses gezeigt werden soll.

\section{4.}

Benjamin hat sein autobiographisches Projekt in erkennbarer Auseinandersetzung mit Marcel Proust unternommen, die über mehrere Jahre intensiv anhielt. Nicht nur hat Benjamin zusammen mit Franz Hessel in den Jahren zwischen 1926 und 1930 einige Bände der Recherche aus dem Französischen übersetzt, sondern er hält schon 1926 brieflich fest, dass die Nähe zu Proust bei ihm »innere Vergiftungserscheinungen« hervorrufe (GB III, 195). Noch 1929 wähnt er sich der Sache so nahe, dass er sich zu keiner Deutung seines Werkes im Stande sieht (GB III, 432). Ein erster Zugang erschließt sich ihm gleichwohl schon 1929 und führt zur Publikation des Essays Zum Bilde Prousts (GS II, 310-324). Im selben Jahr hält Benjamin in Paris, die Stadt im Spiegel fest, dass bei Proust eine regelrechte "unendliche Erinnerung der Erinnerung" am Werk sei (GS IV 359). Im Hinblick auf Prousts Schreibexzess kolportiert er auch die Geschichte, wonach Proust jedes Mal, wenn er von den Setzern die Druckfahnen zur Durchsicht erhielt, diese nicht korrigiert, sondern randvoll mit neuem Text beschrieben den zunehmend verzweifelten Setzern zurückgeschickt hat (GS II, 311f.). "Erinnerung aber ist prinzipiell unabschließbar", ist in Notizen aus derselben Zeit nachzulesen, die Benjamin wohl für einen zweiten Proust-Essay verwenden wollte und die als Proust-Papiere bekannt sind (GS II, 1056). Das Problem, das Benjamin in der Folge weiter beschäftigt und das auch im Zentrum der Aufzeichnung Kleine Rede über Proust aus dem Jahre 1932 steht, dreht sich um das Konzept der mémoire involontaire (GS II, 1064), das mit der Abschließbarkeit des Erinnerungswerkes und also mit seiner Einheit eng verknüpft ist. In der Berliner Chronik bleibt diese Problematik im Frühjahr 1932 für Benjamin virulent, als er die Entstehung seiner eigenen Erinnerungen in der Nachfolge Prousts inszeniert (GS VI, 467). Ähnlich wie bei Proust stehen bei Benjamin Kindheitserinnerungen im Zeichen von Unabschließbarkeit und Uneinheitlichkeit. 


\section{DAVIDE GIURIATO}

In Benjamins fortschreitender Arbeit an seinen Kindheitserinnerungen ist zu beobachten, dass er eine immer größer werdende Distanz zu Prousts mémoire involontaire einnimmt, die schließlich 1938/1939 im Eassy Über einige Motive über Baudelaire zur Einschätzung führt, dass Prousts Werk darauf ziele, „die Figur des Erzählers zu restaurieren«(GS I, 611). Für Benjamin selbst geht diese fortschreitende Distanzierung Hand in Hand mit dem Vorhaben, das unwillkürliche Moment der Erinnerungstätigkeit um ein konstruktives Moment zu erweitern. ${ }^{22}$ Mithin bestimmt diese konzeptuelle Revision auch die Schreibverfahren des eigenen Erinnerungstextes: Benjamin schreibt seine Kindheitserinnerungen nicht kumulativ weiter, sondern er schreibt sie stets um, ohne dass die Erinnerungstexte deswegen abschließbarer würden. Anders als Proust fügt er aber dem Geschriebenen nicht stetig etwas hinzu, sondern verhält sich kritisch revidierend und tendenziell sogar kürzend zu ihm.

Gerade die Umschrift Ausgraben und Erinnern markiert in der Geschichte von Benjamins Proust-Rezeption einen erstmals erkennbaren Abstand zu Proust, der somit ziemlich genau auf August/September 1932 datiert werden kann. Benjamin wirft nämlich in dieser Aufzeichnung erstmals die Frage nach der Methode der Erinnerungstätigkeit auf, indem er ein planmäßiges, willkürliches Vorgehen des Erinnerns diskutiert. Dies fällt umso mehr auf, als die entsprechende Passage in der Berliner Chronik noch nicht zu finden ist. Hier steht das "vergebliche Suchen« der Erinnerung (GS VI, 487) ganz nach der Vorstellung von der mémoire involontaire im Vordergrund. Eine Problematisierung dieses Konzepts findet erst bei der Abschrift statt: Im Schlussteil von Ausgraben und Erinnern formuliert Benjamin eine Anleitung zum Vorgehen "wahrhafte[r] Erinnerungen" (GS IV, 400). Zudem fällt in der Handschrift auf, dass diese Stelle eine markante Umarbeitung enthält:

So müssen wahrhafte Erinnerungen viel weniger berichtend verfahren als genau an dem der Forscher ihrer habhaft wurde.

den Ort bezeichnen, von dem aus die Erinnerung habhaft empfangen wurde.

Das ist die einzige auffällige Streichung in Ausgraben und Erinnern, die in der Edition der Gesammelten Schriften nicht einmal im Apparat wiedergegeben oder erwähnt wird. Gerade an der Streichung kann aber Benjamins konzeptuelle Arbeit rekonstruiert werden, denn sie macht deutlich, dass die Erinnerung für Benjamin nicht mehr etwas ist, das man mit Glück unwillkürlich noch empfangen könnte, sondern dass das Schreiben der Erinnerung 
zuallererst das eigene Vorgehen zu erinnern hat. In der Streichung wird erkennbar, dass Prousts mémoire involontaire von Benjamin als Königsweg zu einer gelingenden Erinnerung gestrichen, aber nicht in ihrer Wirksamkeit negiert, sondern vielmehr einer methodisch und willkürlich vorgehenden Erinnerung integriert wird. Ob die Erinnerungen nun mehr oder weniger zufällig in die Hände desjenigen gelangen, der nach ihnen forscht, stets muss die "wirkliche Erinnerung ein Bild zugleich von dem der sich erinnert geben" (GS IV, 401), stets ist das Schreiben selbst für die Tätigkeit des Erinnernden bestimmend. Die Erinnerung ist hier tatsächlich, wie Rainer Warning in einem erhellenden Aufsatz zu Prousts Textgenese festgehalten hat, das "prekäre Produkt von Nach-Schriften und UmSchriften, die über keinen verläßlichen Ursprung verfügen. ${ }^{23}$ Indem der Erinnerungstext stets umgeschrieben wird, ist gesagt, dass er auch nie richtig geschrieben ist. Und dies gilt umso mehr für Benjamins Kindheitserinnerungen und für die Umschrift Ausgraben und Erinnern, von der schon gesagt wurde, dass sie als provisorischer Entwurf zu betrachten ist. Mit ihr ist auch die Berliner Kindheit als ein Konvolut von Nachschriften und Entwürfen einzuschätzen, die von Benjamin stets umgeschrieben werden, weil sie nie ganz richtig geschrieben sind. Es gibt demnach keinen Grund, Benjamins Streichungen nicht zu berücksichtigen, indem man seine Textentwürfe etwa editorisch zu fertigen Texten bereinigt. Die Durchstreichungen sind vielmehr integraler Bestandteil seines Schreibens.

\section{5.}

Ausgraben und Erinnern macht gerade als Umschrift deutlich, dass das von ihr thematisierte und konzeptualisierte Umwühlen im Erdreich des Gedächtnisses den Text und seine Entstehung selbst betrifft. Als kleiner Erinnerungstext entstanden, der sich nicht an erinnerte Sachverhalte im Sinne erzählbarer Ereignisse orientiert, sondern der sich über das Vorgehen des Erinnerns Klarheit zu verschaffen versucht, kommt sein Schreiber auf ihn zurück, um ihn ab- und umzuschreiben. Im Bild des forschenden Mannes, "der gräbt«, wird zudem deutlich, dass der Entwurf die eigene Textgenese als archäologisches Verfahren figuriert: Dass das deutsche Wort graben und das griechische graphein, das eben sschreiben, gravieren bedeutet, eine etymologische Verwandtschaft besitzen, unterstreicht in Benjamins Metaphorik die Koinzidenz von Erinnern und Schreiben.

23 Rainer Warning: „Vergessen, Verdrängen und Erinnern in Prousts A la recherche du temps perdu«, in: Anselm Haverkamp/Renate Lachmann (Hg.): Memoria - vergessen und erinnern, München (Fink) 1993, S. 160-194. 


\section{DAVIDE GIURIATO}

Erst der Rückgriff auf die Materialität des Überlieferten ermöglicht eine solche Lektüre. Im Falle von Benjamins nachgelassenen Schriften sollte der Literaturforscher gewissermaBen wie in einem guten archäologischen Bericht genau den Ort bezeichnen, an dem er des Dokuments habhaft wurde. Er wird dabei keine fertigen Texte, sondern allenfalls »Torsi« oder Bruchstücke von Texten finden. Benjamins kleine Theorie der Überlieferung und ihre Betonung von materieller Inventarisierung und Dokumentation machen klar, dass es nicht darum gehen kann, diese Fundstücke zu restaurieren, indem man sie etwa zu fertigen Texten herrichtet. Der überlieferungskritische Umgang, der sich an dieser reflektierten positivistischen Methode orientiert und der jenseits eines werkästhetischen Korsetts auf die Dokumentation der Entstehungsprozesse und der archivarischen Materialität gerade nicht verzichtet, wird ganz neue Schichten in Benjamins Schriften entdecken. 